\title{
Giant pulses in pulsar PSR B0031-07
}

\author{
A. D. Kuzmin ${ }^{1,2}$ and A. A. Ershov ${ }^{1,2}$ \\ 1 Pushchino Radio Astronomy Observatory, Astro Space Center, Lebedev Physical Institute, Russian Academy of Sciences, \\ Pushchino 142290, Russia \\ e-mail: akuzmin@prao.psn.ru \\ 2 Isaac Newton Institute, Chile, Pushchino Branch, Russia
}

Received 19 December 2003 / Accepted 15 July 2004

\begin{abstract}
We report on observations of the recently detected (Kuzmin et al. 2004) giant pulses (GPs) from the pulsar PSR B0031-07 at 40 and $111 \mathrm{MHz}$. At $40 \mathrm{MHz}$ the peak flux density of the strongest pulse is $1100 \mathrm{Jy}$, which is 400 times as high as the peak flux density of the average pulse (AP). A pulse whose observed peak flux exceeded the peak of the AP by more than a factor of 200 is encountered approximately once in 800 observed periods. Peak flux density of the GPs compared to the AP peak flux density $S_{\text {peak }}^{\mathrm{GP}} / S_{\text {peak }}^{\mathrm{AP}}$ has roughly a power-law distribution with a slope of -4.5 . GPs at $40 \mathrm{MHz}$ are essentially stronger than those ones at $111 \mathrm{MHz}$. This excess is approximately in inverse proportion to the frequency ratio. The giant pulses are much narrower than the AP, and cluster in two narrow regions of the AP near the peaks of the two components of the AP. Some of the GPs emit at both phases and are double. The separation of the double GP emission regions depends on frequency. Similarly to the frequency dependence of the width of the AP, it is less at $111 \mathrm{MHz}$ than at $40 \mathrm{MHz}$. This suggests that GPs are emitted from the same region of the magnetosphere as the AP, that is in a hollow cone over the polar cap instead of the light cylinder region. PSR B0031-07 as well as the previously detected PSR B1112+50 are the first pulsars with GPs that do not have a high magnetic field at the light cylinder. One may suggest that there are two classes of GPs, one associated with high-energy emission from outer gaps, the other associated with polar radio emission. The GPs of PSR B0031-07 and PSR B1112+50 are of the second class. The dispersion measure $D M$ is found to be $10.900 \pm 0.003 \mathrm{pc} \mathrm{cm}^{-3}$.
\end{abstract}

Key words. stars: neutron - stars: pulsars: general - stars: pulsars: individual: PSR B0031-07

\section{Introduction}

Giant pulses (GPs) are short-duration burst-like sudden increases of intensity of individual radio pulses from pulsars. This rare phenomenon has been detected previously only in five pulsars out of more than 1500 known ones: the Crab pulsar PSR B0531+21 (Staelin \& Sutton 1970), PSR B1112+50 (Ershov \& Kuzmin 2003), the millisecond pulsars PSR B1937+21 (Wolszczan et al. 1984) and PSR B1821-24 (Romani \& Johnston 2001), and the extragalactic pulsar PSR B0540-69 (Johnston \& Romani 2003).

The intensities of the GPs are extremely high. The brightness temperature of GPs of the millisecond pulsar PSR B1937+21 is as high as $T_{\mathrm{B}} \approx 10^{35} \mathrm{~K}$ (Kuzmin \& Losovsky 2002), and $T_{\mathrm{B}} \geq 10^{36} \mathrm{~K}$ for GPs of the Crab pulsar (Kostyuk et al. 2003; Hankins et al. 2003).

Four of these pulsars belong to the group of pulsars with the strongest magnetic field at the light cylinder. Pulsar PSR B1112+50 is the first exception from this group, being a pulsar that exhibits the characteristic features of GPs but has an ordinary value of the magnetic field on the light cylinder. Kuzmin et al. (2004) recently reported the detection of giant pulses from another ordinary magnetic field pulsar, PSR B0031-07. They find that at $111 \mathrm{MHz}$ the peak flux density of the strongest GP is $530 \mathrm{Jy}$, which is 120 times as high as than the peak flux density of the average profile (AP). A pulse whose observed peak fluxes exceed the peak of the AP by more than a factor of 50 is encountered approximately once in 250 observed periods. Giant pulses cluster in a narrow region near the peak of the first component of the AP.

In this paper we report observations of GPs of pulsar PSR B0031-07 at the second frequency of $40 \mathrm{MHz}$, additional observations at $111 \mathrm{MHz}$ and a comparative analysis of the two-frequency data.

\section{Observations and data reduction}

Observations were carried out at two frequencies, 40 and $111 \mathrm{MHz}$.

At $40 \mathrm{MHz}$ observations were performed from August 28 through October 13, 2003 with the DKR Radio Telescope at Pushchino Radio Astronomy Observatory of the Lebedev Physical Institute. The DKR telescope has an effective area of about 8000 square meters. One linear polarization was received. We used a 128-channel receiver with channel bandwidth $1.25 \mathrm{kHz}$. The frequency of the first (highest frequency) channel was $40.982 \mathrm{MHz}$, the sampling interval was $0.819 \mathrm{~ms}$, 
and the time constant was $\tau=1 \mathrm{~ms}$. The duration of each observation was about 16 min (994 pulsar periods). A total of 16 observations containing 15904 pulsar periods was carried out.

From June 21 through September 22, 2003 we performed an additional 16 observations at $111 \mathrm{MHz}$ and 12 simultaneous observations at 111 and $40 \mathrm{MHz}$. At $111 \mathrm{MHz}$ we used the Large Phased Array (BSA) Radio Telescope at Pushchino Radio Astronomy Observatory with an effective area of about 20000 square meters. One linear polarization was received. At both frequencies we used two 64-channel receivers with channel bandwidth $20 \mathrm{kHz}$. The sampling interval was $2.560 \mathrm{~ms}$ and the time constant was $\tau=3 \mathrm{~ms}$. The durations of the observations were about $3 \mathrm{~min}$ (205 pulsar periods) and $9 \mathrm{~min}$ (600 pulsar periods) at 111 and $40 \mathrm{MHz}$ respectively.

All observations were time-referenced to the Observatory rubidium master clock, which in turn was monitored against the National Time standard via TV timing signals. Pulsar ephemerides were obtained from the catalogue by Taylor et al. (1995).

During the off-line data reduction the signal records were cleaned of radio interferences. Subsequently the inter-channel dispersion delays imposed by an interstellar medium were removed. Each observing period was analyzed for pulses with amplitude exceeding a preset level and its amplitude, pulse width and phase were derived.

\section{Results}

At $40 \mathrm{MHz}$ the 212 pulses whose observed peak fluxes density exceeded the peak flux density of the average profile (AP) (which is equivalent to the average pulse) by more than a factor of 100 were selected and analyzed. 19 of them exceeded the peak of the AP by more than a factor of 200. A pulse whose observed peak fluxes exceeded the peak of the AP by more than a factor of 100 is encountered approximately once in 75 observed periods.

Figure 1a shows the strongest observed GP (bold line) together with the AP for all 16 days of observations. The observed peak flux density of this GP exceeds the peak flux density of the AP by factor of 400 . The plot of the AP is presented on a 200 times larger scale and flux densities of the observed GP and AP are shown separately on the left and right sides of the " $y$ " scales.

The value of the GP flux density was determined relative to the known AP flux density as

$S_{\text {peak }}^{\mathrm{GP}}=S_{\text {peak }}^{\mathrm{AP}} \times\left(I^{\mathrm{GP}} / I^{\mathrm{AP}}\right)$,

where $I^{\mathrm{GP}} / I^{\mathrm{AP}}$ is the ratio of the GP to AP peak intensity and $S_{\text {peak }}^{\mathrm{AP}}$ is the peak flux density of AP

$S_{\text {peak }}^{\mathrm{AP}}=S_{\text {mean }}^{\mathrm{AP}} / k_{\text {form }}$,

where $S_{\text {mean }}^{\mathrm{AP}}$ is the flux density averaged over a pulsar period, $k_{\text {form }}$ is the duty cycle of the pulsar.

The value of the AP flux density $S_{\text {mean }}^{\mathrm{AP}}=490 \mathrm{mJy}$ was obtained from Izvekova et al. (1981). The reduction factor is $k_{\text {form }}=0.18$, so the AP peak flux density is $2.7 \mathrm{Jy}$. The strongest

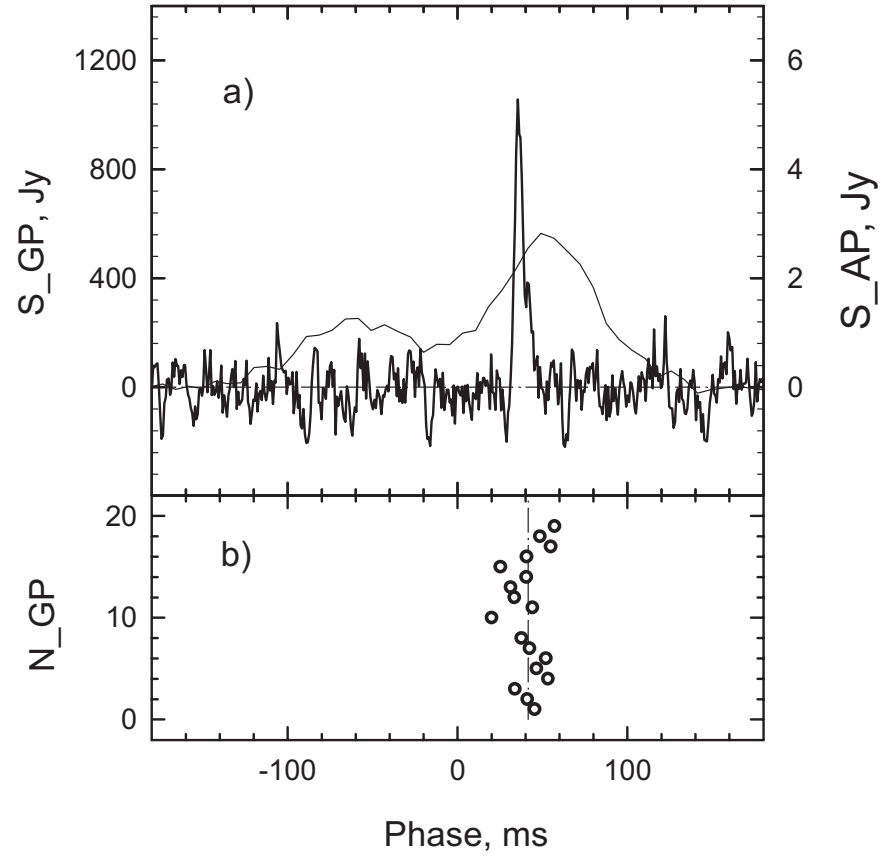

Fig. 1. a) The strongest observed GP at $40 \mathrm{MHz}$ (bold line) together with the AP (thin line). The plot of the AP is presented on a 200 times larger scale and flux densities of the observed GP and AP are shown separately on the left and right sides of the " $y$ " scales. b) The phases of the observed GPs relative to the center of the AP determined as the mid-point between 0.1 intensity levels.

observed GP has $I^{\mathrm{GP}} / I^{\mathrm{AP}}=400$. Then, the observed GP peak flux density is $S_{\text {peak }}^{\mathrm{GP}}=2.7 \times 400=1100 \mathrm{Jy}$. The intrinsic fine structure of the pulses is masked by interstellar scattering. In our measurements of the 19 strongest GPs at $40.9 \mathrm{MHz}$ we derived $\tau_{\mathrm{sc}}=3.1 \pm 0.4 \mathrm{~ms}$. Scattering increases the pulse width and decreases the pulse amplitude. Thus the intrinsic GP peak flux density is $S_{\text {peak }}^{\mathrm{GP}}>1100 \mathrm{Jy}$.

In Fig. 2a we show the cumulative distribution of the ratio of the observed GP peak flux density to the AP peak flux density $S_{\text {peak }}^{\mathrm{GP}} / S_{\text {peak }}^{\mathrm{AP}}$ of all pulses that we have selected and analyzed. In a detectable interval of $S_{\text {peak }}^{\mathrm{GP}} / S_{\text {peak }}^{\mathrm{AP}}$ from 100 to 400 , the histogram has roughly a power-law distribution with a slope of -4.5. Since for $S_{\text {peak }}^{\mathrm{GP}} / S_{\text {peak }}^{\mathrm{AP}}<100$ the pulse signal-to-noise ratio is less than 5 , at lower intensities the observed distribution is masked by noise.

The distribution for $111 \mathrm{MHz}$, which we have obtained from the Kuzmin et al. (2004) data and additional observations, is shown in Fig. 2b. In a detectable interval of $S_{\text {peak }}^{\mathrm{GP}} / S_{\text {peak }}^{\mathrm{AP}}$ from 30 to 100 , the histogram has roughly a power-law distribution with a slope of -4.8 . Since for $S_{\text {peak }}^{\mathrm{GP}} / S_{\text {peak }}^{\mathrm{AP}}<20$ the pulse signal-to-noise ratio is less than 5 , at lower intensities the observed distribution is masked by noise.

The distributions in Fig. 2 demonstrate, that GPs at $40 \mathrm{MHz}$ are essentially stronger than those at $111 \mathrm{MHz}$. At $40 \mathrm{MHz}$ one in every thousand pulses exceeds the AP by more than a factor of 170 , but at $111 \mathrm{MHz}$ only by 60 . The strongest observed GP at $40 \mathrm{MHz}$ exceeds the peak flux density of the AP by a factor of 400 , but at $111 \mathrm{MHz}$ only by a factor of 120 . In a quantitative 


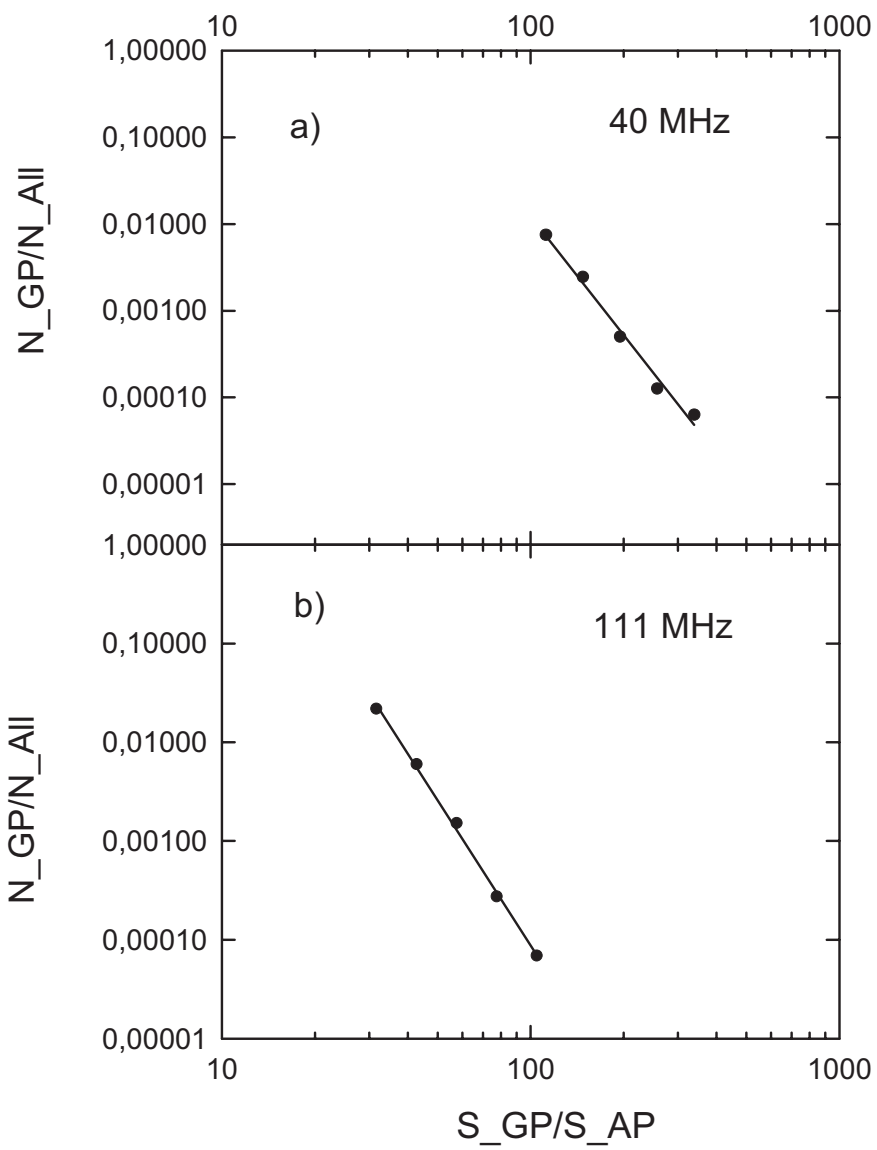

Fig. 2. a) The cumulative distribution of the observed GP peak flux density as related to the AP peak flux density for $40 \mathrm{MHz}$; b) the same for $111 \mathrm{MHz}$.

sense this gain is approximately in inverse proportion to the ratio of frequencies.

From Fig. 2 one can see that the rate of GPs depends on the frequency. At $40 \mathrm{MHz}$ a pulse whose observed peak flux exceeds the peak of AP by more than a factor 100 is encountered once in 85 observed periods, but at $111 \mathrm{MHz}$ only once in 11000 . This may be a reason why there are no claims of GPs from this pulsar at higher frequencies.

Our distributions cover a smaller number of observed pulsar periods (by two orders of magnitude) compared to such histograms for previously known short period pulsars. Therefore, we consider the slope index as a tentative one which needs to be refined.

The mean observed width ${ }^{1}$ of 19 GPs with $S_{\text {peak }}^{\mathrm{GP}} / S_{\text {peak }}^{\mathrm{AP}}>$ 200 is $w_{50}^{\text {obs }}=6 \pm 2 \mathrm{~ms}$. The width of the $\mathrm{AP}^{2}$ for all 12 days of observations is $w_{50}^{\mathrm{AP}}=180 \mathrm{~ms}$. Thus, at $40 \mathrm{MHz}$ GPs are narrower than the AP by about a factor of 30 .

The positions of the GPs are stable within the AP and they cluster in a narrow window near the maximum of the second component of the AP. Figure $1 \mathrm{~b}$ shows the phases $\Phi$ of the GPs which exceeded the AP by factor of 100 . The average phase difference between the GPs and the center of the AP is

\footnotetext{
1 The width is not corrected for scatter and dispersion broadening.

2 The width of the AP was measured at half the maximum height of the leading and trailing components.
}

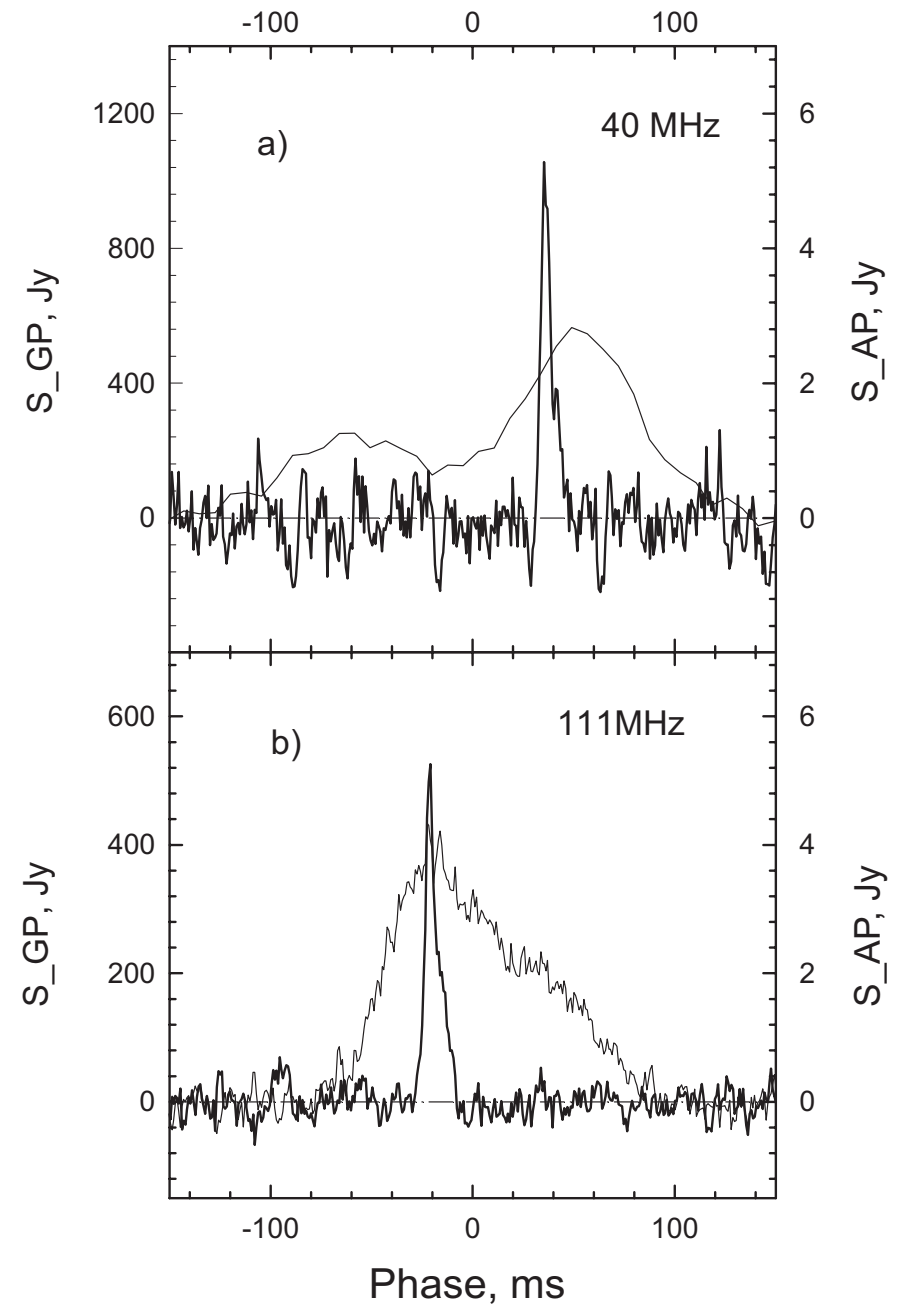

Fig. 3. a) The strongest observed GP at $40 \mathrm{MHz}$ (bold line), together with the AP (thin line); b) the strongest observed GP at $111 \mathrm{MHz}$ (bold line), together with the AP (thin line). Plots of GPs and APs are presented on different scales, shown on the left and right sides of the " $y$ " axis.

$\Phi_{40} \cong 40 \mathrm{~ms}$. GPs cluster in a narrow phase window $\Delta \Phi=$ $\pm 10 \mathrm{~ms}$. The clustering is tighter for stronger GPs.

The GPs are not identified with subpulses, since contrary to the stable phase position of GPs, the phase position of subpulses drift across the AP. GPs are also narrower than the subpulses of this pulsar $w_{50}=15 \mathrm{~ms}$ at 60 and $102 \mathrm{MHz}$ (Izvekova et al. 1993).

The GP brightness temperature is

$T_{\mathrm{B}}=S \lambda^{2} / 2 k \Omega$.

Here $\lambda$ is the radio wavelength, $k$ is the Boltzmann constant, and $\Omega$ is the solid angle of the radio emission region. Adopting $\Omega \simeq(l / d)^{2}$, where $l$ is the size of the radio emission region and $d$ the distance to the pulsar, and further adopting $l \leq c \times w_{50}$, where $c$ is the speed of light and the distance to the pulsar $d=$ $0.68 \mathrm{kpc}$ (Taylor et al. 1995), we obtain $T_{\mathrm{B}} \geq 10^{28} \mathrm{~K}$.

The most unexpected and interesting feature of our observations at $40 \mathrm{MHz}$ is the non-coincidence of the phase positions of the GPs at 40 and $111 \mathrm{MHz}$. In Fig. 3 we present the time-alignment of GPs and APs at 40 and $111 \mathrm{MHz}$ 
(Kuzmin et al. 2004). One can see that the GP at $40 \mathrm{MHz}$ is separated from the GP at $111 \mathrm{MHz}$ by about $60 \mathrm{~ms}$ and that they are located in two different parts of the AP. At $111 \mathrm{MHz}$ the GP is located near the maximum of the first component of the AP, whereas at $40 \mathrm{MHz}$ the GP is located near the maximum of the second component.

To verify this peculiarity, we have performed a control determination of the dispersion measure $D M$. For this we varied the value of $D M$ by a process of removal of the interchannel dispersion delays and searched their optimal value, which provides the maximum amplitude of individual pulses. We processed 440 individual pulses at $111 \mathrm{MHz}$ and 337 at $40 \mathrm{MHz}$. The mean value of dispersion measure was obtained to be $D M=10.900 \pm 0.003 \mathrm{pc} \mathrm{cm}^{-3}$. This value is consistent with the catalog value $10.89 \pm 0.01 \mathrm{pc} \mathrm{cm}^{-3}$ (Taylor et al. 1995) and confirms the validity of the non-coincidence of the phase positions of the GPs at 40 and $111 \mathrm{MHz}$.

One may suggest two explanations of such behavior. The first is that the emission region of GPs moves with frequency. The second proposes that there are two separate phase emission regions for the GPs. In this case, one may expect that some of the GPs will emit at both phases and are double.

To clarify this point, we undertook a more detailed inspection of the GPs. Our first-line reduction program searched only for a single GP per pulsar period, whose amplitude exceeded a preset level. However, one may expect that, in the case of two emission regions, some GPs have two components, where the first component is observed mainly at $111 \mathrm{MHz}$ and the second at $40 \mathrm{MHz}$. Therefore we performed an addition search of the observed GPs for two-component structure. For this search we used a $20 \mathrm{kHz}$ channel bandwidth at both frequencies. The answer is yes: - some GPs, both at 40 and $111 \mathrm{MHz}$, have a two-component structure. We detected 12 double GPs whose amplitude exceeded the AP by a factor of 50 at $111 \mathrm{MHz}$ and 5 double GPs with amplitude exceeding AP by a factor of 20 at $40 \mathrm{MHz}$.

An example of two-component GPs is shown in Fig. 4. Since at $40 \mathrm{MHz}$ double pulses were searched in a $20 \mathrm{kHz}$ channel bandwidth, the dispersion pulse broadening of $30 \mathrm{~ms}$ reduces the strength of the GP and distorts the observed pulse shape compared to other GPs, which were observed in $1.25 \mathrm{kHz}$ channel bandwidth.

The phases of these double GPs, as well as single GPs from our observations and the Kuzmin et al. (2004) data are plotted in Fig. 5 together with APs.

One can see that the phases of the trailing component of double GPs at $40 \mathrm{MHz}$ (Fig. 5c) fit the phases of the single GPs at this frequency (Fig. 5b). In a similar manner, the phases of the leading component of double GPs at $111 \mathrm{MHz}$ (Fig. 5d) fit the phases of the single GPs at $111 \mathrm{MHz}$ (Fig. 5e). This supports the concept of two emission regions of GPs. The first dominates at $111 \mathrm{MHz}$, the second dominates at $40 \mathrm{MHz}$ and both form double GPs.

Twelve observation sessions, containing 2460 pulsar periods, were performed simultaneously at 40 and $111 \mathrm{MHz}$. We also used the $20 \mathrm{kHz}$ channel bandwidth at both frequencies. Forty pulses at $40 \mathrm{MHz}$ and 45 pulses at $111 \mathrm{MHz}$ with peak intensities exceeding the peak intensity of the AP by more than a

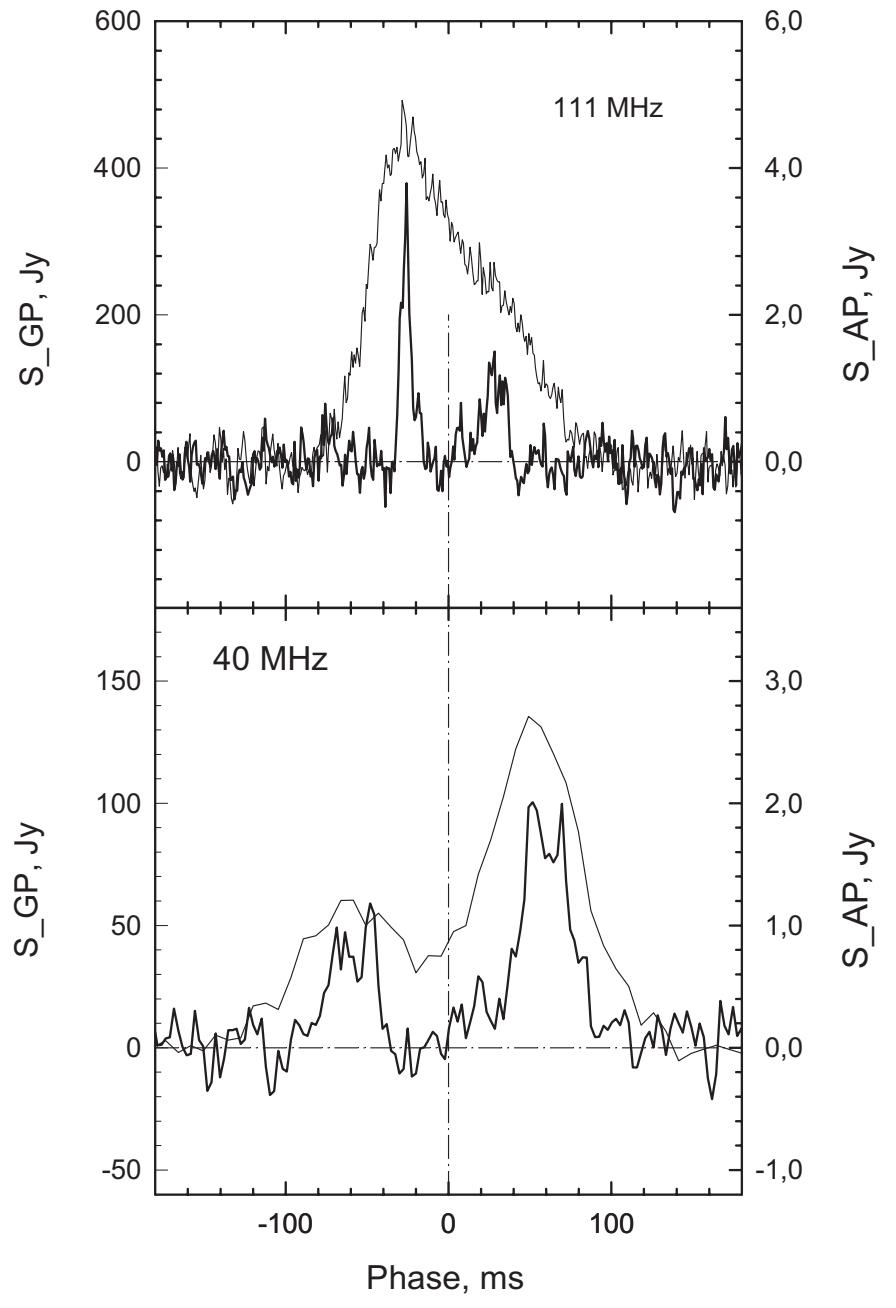

Fig. 4. a) The double GP (bold line) observed at $111 \mathrm{MHz}$ together with the AP (thin line); b) the double GP (bold line), observed at $40 \mathrm{MHz}$, together with the AP (thin line).

factor of 20 were selected and analyzed. Only two among these pulses were found in the same pulse phase inside the same pulsar period and identified as simultaneous. The probability that there are accidental coincidences of GPs simultaneously at two frequencies is equal to $P_{40} * P_{111}=40 / 2460 * 45 / 2460 \approx 10^{-7}$; this is significantly lower than the observed detection rate of simultaneous GPs of $2 / 2460 \approx 10^{-3}$.

The measured spectral indices between frequencies of 40 and $111 \mathrm{MHz}$ for these 2 simultaneous GPs are $\alpha^{\mathrm{GP}}=-0.6$ and -1.1 , corresponding to the low-frequency turn-over region of this pulsar (the spectral index of the AP is $\alpha^{\mathrm{AP}}=-0.2$ ). However, only a small number of simultaneous GPs was observed. This possibility therefore needs further study.

\section{Discussion}

The GPs that we detected from PSR B0031-07 exhibit all characteristic features of the classical GPs from PSR B0531+21 and PSR B1937+21.

The peak intensities of the GPs exceed the peak intensity of the AP by more than a factor of 400. The histograms of the flux density have a power-law distribution. The GPs are 


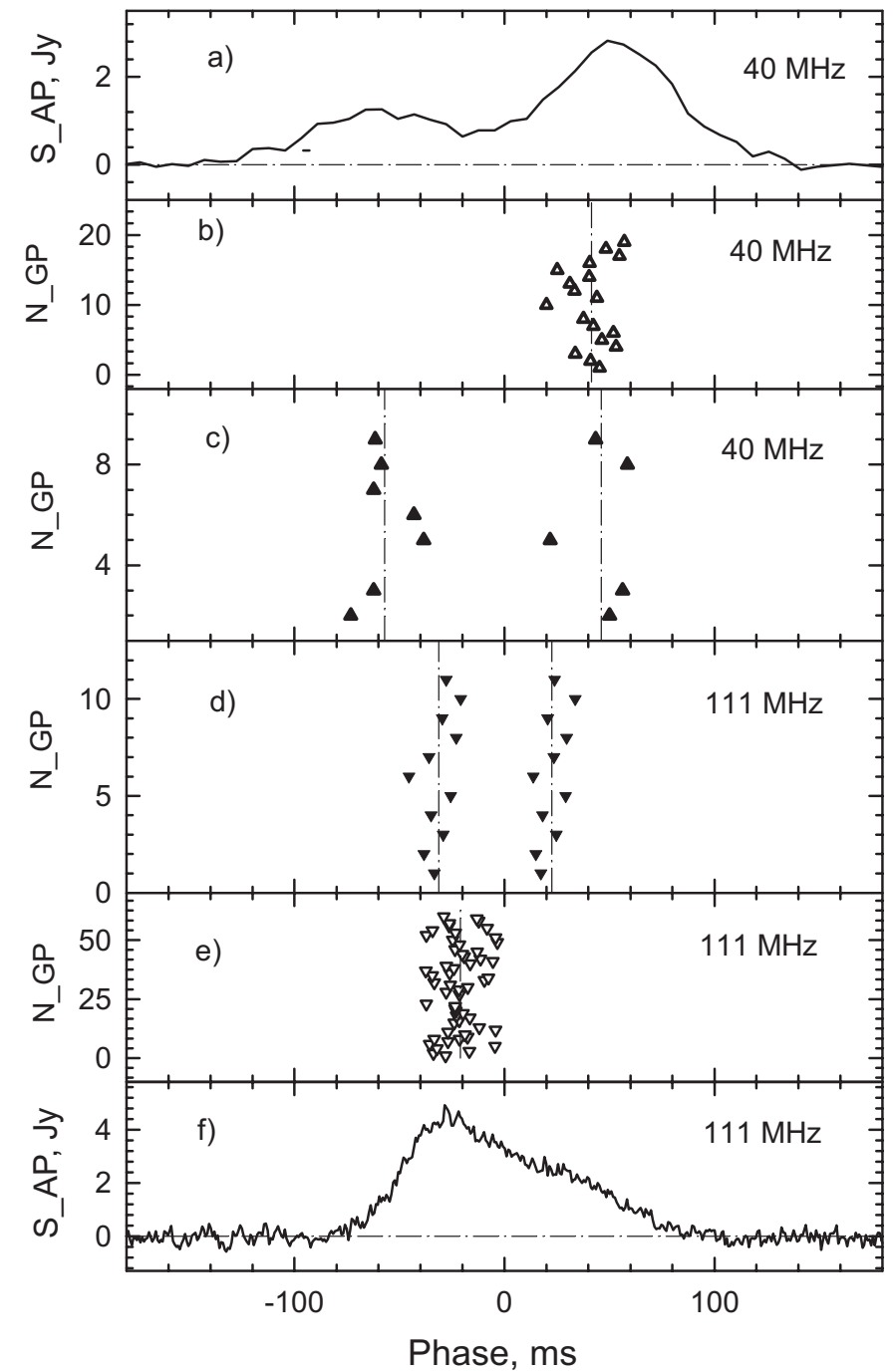

Fig. 5. a) Integrated profile at $40 \mathrm{MHz}$; b) phases of the observed single GPs at $40 \mathrm{MHz}$; c) phases of the observed double GPs at $40 \mathrm{MHz}$; d) phases of the observed double GPs at $111 \mathrm{MHz}$; e) phases of the observed single GPs at $111 \mathrm{MHz}$; f) integrated profile at $111 \mathrm{MHz}$.

much narrower than the AP and their phases are stable inside the integrated profile.

Alongside these similarities, the GPs of the pulsar PSR B0031-07 have noticeable differences. Previously known pulsars with GPs belong to the group of pulsars with very high magnetic field on the light cylinder. Pulsar PSR B0031-07, as well as the previously detected PSR B1112+50 (Ershov \& Kuzmin 2003), are the first ones with ordinary magnetic fields on the light cylinder.

The most unexpected and interesting result is the clustering of the GPs in two different regions. This indicates that there are two emission regions of GPs. The separation of these regions at $40 \mathrm{MHz}$ is larger than at $111 \mathrm{MHz}$; this is corresponds to the increase in the width of the AP, which is interpreted as a divergence of the magnetic field lines in the hollow cone model of pulsar radio emission. This suggests that the GPs from this pulsar, and possibly from PSR B1112+50, originate in the same region as the AP, that is in a hollow cone over the polar cap instead of in the light cylinder region.
One may suggest that there are two classes of GPs, one associated with high-energy emission from the outer gaps, the other associated with polar radio emission. The GPs of PSR B0031-07 and PSR B1112+50 are of the second class.

\section{Conclusions}

The detection of giant pulses from pulsar PSR B0031-07 has been confirmed. The peak flux density of the strongest pulse is $1100 \mathrm{Jy}$, which is 400 times as high as than the peak flux density of the average profile.

The GPs are much narrower than the average profile and cluster in two narrow regions of the average profile. The separation of the two GP emission regions decreases with frequency. This suggests that the GPs from this pulsar are emitted from the same region as the AP, that is in the hollow polar cone instead of in the light cylinder region.

Pulsar PSR B0031-07, as well as the previously detected PSR B1112+50, are the first pulsars with GPs that do not have a high magnetic field at the light cylinder, such as previously known pulsars with GPs have. They may be a separate class of GPs.

The dispersion measure $D M$ is found to be $10.900 \pm$ $0.003 \mathrm{pc} \mathrm{cm}^{-3}$.

Acknowledgements. We wish to thank V. V. Ivanova, K. A. Lapaev \& A. S. Aleksandrov for assistance during observations. We are grateful to Francis Graham Smith for improving the English and valuable comments. We are grateful to the anonymous referee for comments which enhanced the paper. This work was supported in part by the Russian Foundation for Basic Research (project No. 01-02-16326) and the Program of the Presidium of the Russian Academy of Sciences "Non-steady-state Processes in Astronomy."

\section{References}

Ershov, A. A., \& Kuzmin, A. D. 2003, Pis'ma v AZh, 29, 111 (Astron. Lett., 29, 91)

Hankins, T. H., Kern, J. S., Weatherall, J. C., \& Eilek, J. A. 2003 , Nature, 422, 141

Izvekova, V. A., Kuzmin, A. D., Malofeev, V. M., \& Shitov, Yu. P. 1981, Ap\&SS, 78, 45

Izvekova, V. A., Kuzmin, A. D., Lyne, A. G., et al. 1993, MNRAS, 261,865

Johnston, S., \& Romani, R. W. 2003, ApJ, 590, L95

Kostyuk, S. V., Kondratiev, V. I., Kuzmin, A. D., Popov, M. V., \& Soglasnov, V. A. 2003, Pis'ma v AZh, 29, 440 (Astron. Lett., 29, 387)

Kuzmin, A. D., \& Losovsky, B. Ya. 2002, Pis'ma v AZh, 28, 25 (Astron. Lett., 28, 21)

Kuzmin, A. D., Ershov, A. A., \& Losovsky, B. Ya. 2004, Pis'ma v AZh, 30, 285 (Astron. Lett., 30, 247)

Romani, R. W., \& Johnston, S. 2001, ApJ, 557, L97

Staelin, D. H., \& Sutton, J. M. 1970, Nature, 226, 69

Taylor, J. H., Manchester, R. N., Lyne, A. G., et al. 1995, Catalog of 706 Pulsars, unpublished

Wolszczan, A., Cordes, J. M., \& Stinebring, D. R. 1984, in Millisecond Pulsars, ed. S. P. Reynolds \& D. R. Stinebring (NRAO, Green Bank), 63 